\title{
Association of AVPR1A gene microsatellites with Autism Spectrum Disorder in south Indian population
}

\section{Dhanesh Mandamkulathil ${ }^{1 *}$, Anitha Ayyappan Pillai ${ }^{2}$, Suresh A Poovathinal ${ }^{2}$, Shyamala K Chengappa ${ }^{3}$, Adimoolam Chandrasekar ${ }^{4}$, Ashitha Sidappa Niranjana Murthy ${ }^{5}$, Dhurbagula Srividhya ${ }^{3}$, Sridevi Tirumalaraj ${ }^{1}$,Pardhanandana Reddy Pengaluru ${ }^{1}$.}

\footnotetext{
${ }^{1}$ Mahatma Gandhi National Institute of Research \& Social Action, Kimtee colony, Street No. 1, Rd Number 12, Banjara Hills, Hyderabad, Telangana 500034.

${ }^{2}$ Institute for Communicative and Cognitive Neurosciences (ICCONS) Kavalappara, Shoranur, Palakkad 679 523, Kerala, INDIA.

${ }^{3}$ Department of Speech Language Pathology, All India Institute of Speech \& Hearing

Manasagangothri, Mysore 570006

${ }^{4}$ Anthropological Survey of India, Southern Regional Centre, Manav Bhawan 93/12 \& 94/12, Bogadi, Mysore - 570006

${ }^{5}$ Genetics and Genomics Lab, Department of Studies in Genetics and Genomics, University of Mysore, Manasagangotri-06, Karnataka, India

*Corresponding Author: dhaneshmk@hotmail.com, Tel.: 8281687969
}

Available online at: www.isroset.org

Received: 13/Feb/2019, Accepted: 24/Feb/2019, Online: 28/Feb/2019

\begin{abstract}
Pharmacological studies have shown the therapeutic potential of Arginine Vasopressin Receptor (AVPRlA) for Autism Spectrum Disorders (ASDs) with socialization and communication symptoms. Present study investigated the association of promoter microsatellites (RS1 and RS3) polymorphisms of AVPRIA gene with ASDs in south Indian ethnicity. We recruited 225 subjects that constitutes of 118 ASD subjects diagnosed based on DSM-IV criteria and 107 controls, aged between 2 and 25 years of south Indian ethnicity. Case control association with RS1 allele length- 318bp, $(P=0.009)$; allele length- 330bp $(P=0.02)$; overall association with RS1 $(P=0.02)$ and RS3 allele length- 333bp $(P=0.002), 335 \mathrm{bp}(P=0.045)$ over all association $(P=0.002)$ with ASDs was found to be significant. Our results reveal that, RS1 and RS3 microsatellites of AVPRIA gene contribute to the susceptibility of ASD in south Indian ethnicity.
\end{abstract}

Keywords-Autism Spectrum Disorder (ASD), AVPR1A, Allele, Microsatellites.

\section{INTRODUCTION}

Autism spectrum disorders (ASDs), are complex neurodevelopmental disorder which severely affects an individual's social life with communication difficulties, restricted and repetitive behaviors (NIH-National Institute of Mental Health, 2018). In the light of increased incidence of ASD's in the recent decades, the focus of research has now shifted towards ASD. However, the cause of ASD still remains unclear, partly because of its etiological heterogeneity. It is only recently that the effects of vasopressin on neural signals underlying social cognitive processes are being characterized. Oxytocin(OT) and Arginine-Vasopressin(AVP) are molecularly similar neuropeptides that are evolutionarily conserved and have important roles in mammalian social behaviour and cognition [1]. AVPRIA is one of the most extensively studied receptor that mediates social behaviours like aggression, social recognition and paternal behaviour [2] with major effects on memory [3]. Interestingly, the studies in human subjects have revealed a significant association between the autism and heritable genetic defects in AVPRIA [4].

Human AVPRlA gene is located at $12 \mathrm{q} 14-15$ with two coding exons separated by $2.2-\mathrm{kb}$ intron [5] AVPRIA, a $\mathrm{G}$ protein receptor is expressed in the septal nuclei, thalamus and basal nuclei of amygdala in the brain. These neuropeptides are transported through neurosecretory axons to the posterior hypothalamus to function as neurohypophyseal peptides [6] Various studies have established the possible associations between polymorphisms in the promoter region of the AVPRIA gene and ASDs. The human $A V P R 1 A$ promoter region contains two microsatellite repeats, $\mathrm{RS} 1$ and $\mathrm{RS} 3$, in the $5^{\prime}$ flanking region. Of these, 
RS3 is a complex repeat located 3625bp upstream of the transcription start site, and RS1 is a (GATA)n repeat located 553bp upstream of the start site. Different family based studies have shown the significant transmission of RS1 and RS3 microsatellites [7][8][4]. Recently, RS1, RS3, and intronic microsatellite (AVR) showcased the transmission of alleles in Finnish population [9] Shorter repeat alleles of RS1 and RS3 decreased the relative promoter activity in the human neuroblastoma cell line SH-SY5Y[10]

\section{RELATED WORK}

Research conducted on the behaviour of the Prairie vole a commonly-used animal model to study genes involved in affiliative social behaviours provided preliminary clues on the mammalian genetics and neural mechanism of social behaviour. It strongly suggests that regulatory defects of this system might contribute to neurodevelopmental disorders such as ASD [11] Though the expression of AVPRIA is species specific, studies conducted on Prairie voles have revealed that differential expression of proximal 5'-flanking region might result in behaviour changes[12] Furthermore, humanized AVPRIA mice displayed enhanced reciprocal social interactions [13] A group of scientists generated three mouse lines in which the mouse avprla 5'-flanking region was replaced with corresponding sequence from Prairie voles and showed significant contributions of the microsatellite using genome editing in mice [14] As mentioned above, animal model experiments provided prominent supports on the role of $A V P R I A$ gene and promoter microsatellites in the development of ASDs.

Intranasal administration AVP antagonists have probed the effects of AVPRIA signalling on social behaviour. Genetic association studies have inspected the association of variation in the AVPRIA gene and social traits [12] Supporting this evidence, the AVPRlA antagonist, balovaptan, has the potential to be the first pharmacotherapy to help improve core socialization and communication symptoms of ASD. It was granted the 'breakthrough therapy' designation by the Food and Drug Administration and is proceeding to phase II testing supported by Roche, Basel (2018). These findings, together with the known role of AVPRIA in social skill formation, suggest a link between vasopressin dysregulation and development of autism. However the reports of RS1 and RS3 microsatellites association were only available from different ethnicity but none based on Indian ethnicity. Hence we focused our study to identify RS1 and RS3 association with ASDs in the south Indian population.

\section{METHODOLOGY}

\section{Selection of subjects}

We recruited 225subjects, which constituted of 118 ASD subjects (90 Male and 28 females) and 107 controls (56 males and 51 females), between the ages of 2 to 25 years
(Mean age $=7 \pm 10$, standard deviation=4.82 \pm 3.33 ) from south Indian ethnicity as self-reported. The ASD subjects diagnosed by the multidisciplinary team of neurologist, clinical psychologist and speech-language pathologist were by DSM-IV criteria. Fragile $\mathrm{X}$ syndrome and other syndromic forms of ASD are excluded from this study. All participants/guardians received a full explanation of the study protocols and objectives before obtaining their informed and written consent. Ethical clearance for this study was obtained from Mahatma Gandhi National Institute of Research and Social Action (MGNIRSA-affiliated research centre, University of Mysore) and also from ICCONS (Institute of Communicative and Cognitive Neuroscience) Ethical Committee.

\section{Microsatellite selection and Genotyping}

Selected markers (RS1 and RS3) identified as pharmacological importance with ASD and also exhibited importance for replication in different population (Table.1). PCR (Polymerase chain reaction) primers were designed based on previous studies [8] Approximately $5 \mathrm{ml}$ of peripheral blood sample was collected in BD $\mathrm{K}_{2}$ vacutainer ${ }^{\circledR}$ (BD, NJ, USA) containing EDTA as anticoagulant. DNA was extracted by standard phenol chloroform and salting out method. PCR conditions used were denaturation step $95^{\circ} \mathrm{C}-$ $5 \mathrm{~min}, 95^{\circ} \mathrm{C}-30 \mathrm{sec}$, annealing with $55^{\circ} \mathrm{C}-30 \mathrm{sec}, 72^{\circ} \mathrm{C}-40 \mathrm{sec}$ for 30 cycles then Extension $72^{\circ} \mathrm{C}-10 \mathrm{~min}$. Microsatellite peaks were measured on the Applied Biosystems 3730xl and sized with Genemapper 4.0 (AppliedBiosystems ${ }^{\mathrm{TM}}$, Foster City,CA,USA).

\section{Statistical Analysis}

Case control association test for RS1 and RS3 microsatellites were performed using the UNPHASED 3.1.7[15] Power was calculated using online sample size estimator (http://osse.bii.a-star.edu.sg/calculation2.php).

\section{RESULTS AND DISCUSSION}

AVPRIA microsatellites RS1 and RS3 allelic length shows significant association with ASDs. (Table.2 and Table.3) RS1 allele length- 318bp $(P=0.009)$; allele length- 330bp ( $P$ $=0.02)$; and overall association with $\operatorname{RS} 1(P=0.02)$ exhibited a significant association with ASDs. In case of RS3 allele length- 333bp $(P=0.002), 335 \mathrm{bp}(P=0.045)$ and over all association $(P=0.002)$ also showed the same pattern. Allele frequency of RS1-318bp was in cases-0.077 and in control-0.023 but the frequency in RS1-330bp was in cases 0.043 and in control 0.009 . However, in the case of RS3 allelic frequency and count for $333 \mathrm{bp}$ in cases 0.24 controls 0.13 . Similarly in $335 \mathrm{bp}$ in cases -0.017 and in controls is 0.051 (Fig.1 and Fig.2). Power estimated for RS1 is $46.50 \%$ based on MAF (Minor Allele Frequency) for Cases $-7.8 \%$, Control $-2.3 \%$ and significant level $5 \%$. For RS3 power to detect association was $56.00 \%$ based on MAF 
in Cases $24.1 \%$ and control $13.1 \%$ and significant level as $5 \%$.

\section{Discussion}

The present case-control study was designed to identify the association status of popular microsatellites of commonly studied behaviour related gene AVPRIA. It is more strongly implicated in neuropsychiatric phenotypes, specifically ASD. Earlier studies have shown the presence of microsatellite repeats upstream of the gene $A V P R I A$. In humans, the promoter region of the $A V P R 1 A$ includes a number of highly polymorphic microsatellites like RS1 and RS3. Long repeats of AVPRIA RS3 promoter region predicts increased expression in post-mortem hippocampal specimens [16] The mechanism by which the microsatellite allele length actually affects transcription efficacy is not yet known. The current study explores the influence of microsatellite (RS1 and RS3) length variability with occurrence of ASD in south Indian population.

However, this study has limited power and there were no reported case control study on RS1 and RS3 markers with ASD. However, we have adapted a statistical methodology from a study on choral singers and nonmusicians [17]. The literature reported so far has produced inconsistent association with different alleles and markers implicated in AVPRIA gene. In non-clinical studies, it has been identified that RS1 and RS3 alleles of a specific length were highly associated with the social phenotype under investigation. In a study of personality traits, the RS1 320bp target allele mediated the association with harm avoidance and novelty seeking. The carriers also showed significantly lower left amygdala activity relative to the non-carriers[4] For RS3, the presence of a target allele (327-334bp) has been associated with less altruistic behaviour [18].

Latest studies suggest that targeting these elements in arginine vasopressin pathways are possible candidate for drug discovery as well as a source of potential biomarkers. This could help in early diagnosis of social disorders, especially ASDs [19]. Both RS3 and RS1 length variants were found to be associated with restrictive, repetitive behaviours- core feature of ASDs- but not overall diagnosis [20]. Along with all supporting evidence, the present study concludes that association of RS1 and RS3 allelic length variations may be important in the development of ASD.

\section{CONCluSION AND FUTURE SCOPE}

Evidence from both human and animal studies implicated AVPR1A receptor in mediating and modulating key social behaviours that are challenging for children with ASD. No pharmaceutical treatments are currently exists for the better behavioural improvement in ASD. Present study supported the AVPR1A microsatellites (RS1 and RS3) and its association with ASD in south Indian population. However,
Inconsistency in allelic length in different studies may be due to different ethnicity, statistical methodologies and mostly because of the lack of specificity of RS1 and RS3 primers used. Application of this methodology using the same primer sets in similar population may provide better clarity in allelic length in association to RS1 and RS3 markers with ASDs. Thus present findings recommends a replication study for the same markers in a larger case-control sample set after quantifying for various behavioural parameters along with expressive and responsive language levels within the same population which is necessary to prove functional association of AVPRIA microsatellites with ASDs.

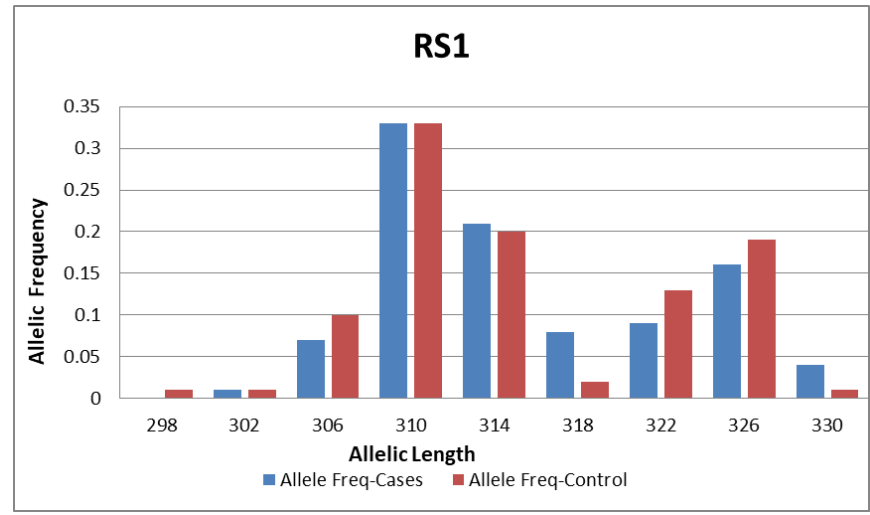

Fig.1: Alleilic frequency of RS1 microsattlites in ASD and control subjects.

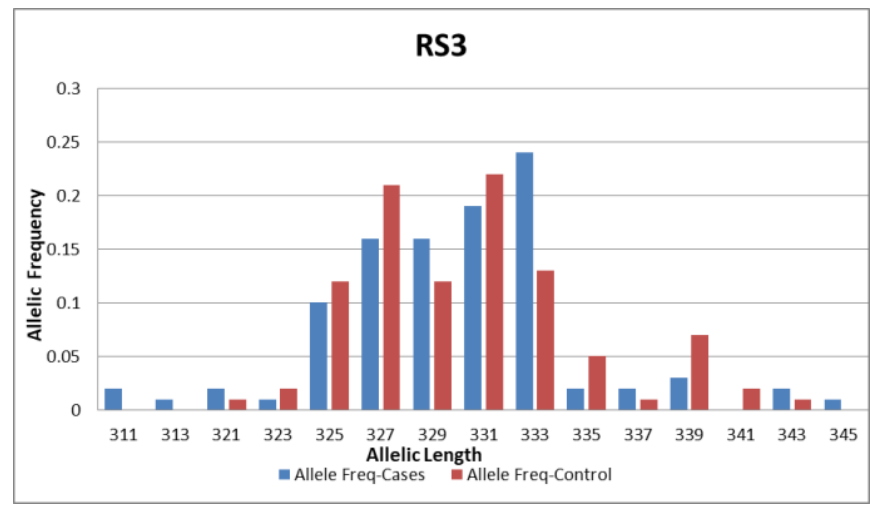

Fig.2: Alleilic frequency of RS3 microsattlite in ASD and control subjects.

\section{CONFLICT OF INTEREST}

For this research paper there is no conflict of interest as confirmed by the authors.

\section{ACKNOWLEDGMENT}

The authors would like to thank Dr. Deverakonda Suresh, Director of MGNIRSA, to facilitate complete support to make successful completion of the work. We thank all the 
participants/care-givers for their cooperation. We also thank ,Dr. Srinivas Naidu Kovali (Scientist-AIISH), Arja Cholendra (PhD) Sudheesh AP (Research Scholar,RGCB)and Mrs.Vijitha Viswambharan (Project fellow, ICCONS).

\section{REFERENCES}

[1] Z. R. Donaldson and L. J. Young, "Oxytocin, vasopressin, and the neurogenetics of sociality.," Science, vol. 322, no. 5903, pp. 900 904, Nov. 2008.

[2] A. D. Hammock and L. J. Young, "Oxytocin, vasopressin and pair bonding: implications for autism.," Philos. Trans. R. Soc. Lond. B. Biol. Sci., vol. 361, no. 1476, pp. 2187-2198, Dec. 2006.

[3] E. B. Keverne and J. P. Curley, "Vasopressin, oxytocin and social behaviour.," Curr. Opin. Neurobiol., vol. 14, no. 6, pp. 777-783, Dec. 2004.

[4] H. Wassink et al., "Examination of AVPR1a as an autism susceptibility gene.," Mol. Psychiatry, vol. 9, no. 10, pp. 968-972, Oct. 2004.

[5] M. Thibonnier, M. K. Graves, M. S. Wagner, C. Auzan, E. Clauser, and H. F. Willard, "Structure, sequence, expression, and chromosomal localization of the human V1a vasopressin receptor gene.," Genomics, vol. 31, no. 3, pp. 327-334, Feb. 1996.

[6] A. Meyer-Lindenberg et al., "Genetic variants in AVPR1A linked to autism predict amygdala activation and personality traits in healthy humans.," Mol. Psychiatry, vol. 14, no. 10, pp. 968-975, Oct. 2009.

[7] S.-J. Kim et al., "Transmission disequilibrium testing of arginine vasopressin receptor 1A (AVPR1A) polymorphisms in autism.," Mol. Psychiatry, vol. 7, no. 5, pp. 503-507, 2002.

[8] N. Yirmiya et al., "Association between the arginine vasopressin 1a receptor (AVPR1a) gene and autism in a family-based study: mediation by socialization skills.," Mol. Psychiatry, vol. 11, no. 5, pp. 488-494, May 2006.

[9] K. Kantojarvi et al., "Association and Promoter Analysis of AVPR1A in Finnish Autism Families.," Autism Res., vol. 8, no. 5, pp. 634-639, Oct. 2015.

[10] K. E. Tansey, M. J. Hill, L. E. Cochrane, M. Gill, R. J. Anney, and L. Gallagher, "Functionality of promoter microsatellites of arginine vasopressin receptor 1A (AVPR1A): implications for autism.," Mol. Autism, vol. 2, no. 1, p. 3, Mar. 2011.

[11] L. A. McGraw and L. J. Young, "The prairie vole: an emerging model organism for understanding the social brain.," Trends Neurosci., vol. 33, no. 2, pp. 103-109, Feb. 2010.

[12] Z. R. Donaldson and L. J. Young, "Oxytocin, vasopressin, and the neurogenetics of sociality.," Science, vol. 322, no. 5903, pp. 900 904, Nov. 2008.

[13] R. Charles et al., "Introduction of the human AVPR1A gene substantially alters brain receptor expression patterns and enhances aspects of social behavior in transgenic mice.," Dis. Model. Mech., vol. 7, no. 8, pp. 1013-1022, Aug. 2014.

[14] J. M. Sadino and Z. R. Donaldson, "Prairie Voles as a Model for Understanding the Genetic and Epigenetic Regulation of Attachment Behaviors.," ACS Chem. Neurosci., vol. 9, no. 8, pp. 1939-1950, Aug. 2018.

[15] F. Dudbridge, "Likelihood-based association analysis for nuclear families and unrelated subjects with missing genotype data.," Hum. Hered., vol. 66, no. 2, pp. 87-98, 2008.

[16] A. Knafo, C. Zahn-Waxler, C. Van Hulle, J. L. Robinson, and S. H. Rhee, "The developmental origins of a disposition toward empathy: Genetic and environmental contributions.," Emotion, vol. 8, no. 6, pp. 737-752, Dec. 2008.
[17] A. P. Morley et al., "AVPR1A and SLC6A4 polymorphisms in choral singers and non-musicians: a gene association study.," PLoS One, vol. 7, no. 2, p. e31763, 2012.

[18] R. Avinun et al., "AVPR1A variant associated with preschoolers' lower altruistic behavior.," PLoS One, vol. 6, no. 9, p. e25274, 2011.

[19] R. Zhang, H.-F. Zhang, J.-S. Han, and S.-P. Han, “Genes Related to Oxytocin and Arginine-Vasopressin Pathways: Associations with Autism Spectrum Disorders.," Neurosci. Bull., vol. 33, no. 2, pp. 238-246, Apr. 2017.

[20] S. M. Francis, S.-J. Kim, E. Kistner-Griffin, S. Guter, E. H. Cook, and S. Jacob, "ASD and Genetic Associations with Receptors for Oxytocin and Vasopressin-AVPR1A, AVPR1B, and OXTR.," Front. Neurosci., vol. 10, p. 516, 2016.

\section{AUTHORS PROFILE}

Mr.Dhanesh Mandamkulathil, Research Scholar Biotechnology MGNIRSA - (University of Mysore) School of Biotechnology Mahatma Gandhi National Institute of Research and Social Action (MGNIRSA) Kimtee colony, Street No. 1, Rd Number 12, Banjara Hills, Hyderabad, Telangana 500034. Email:dhaneshmk@hotmail.com Phone: 08281687969. (Corresponding author )

Dr.Anitha Ayyappan Pillai Ph.D. Associate Professor, Dept. of Neurogenetics Institute for Communicative and Cognitive Neurosciences (ICCONS) Kavalappara, Shoranur, Palakkad 679 523, Kerala, INDIA Mobile: Phone (office): 0466 2224869 email: anitha.a72@gmail.com 8589970421

Dr.Suresh A Poovathinal Director Institute for Communicative and Cognitive Neurosciences (ICCONS), Kavalappara, Shoranur, Palakkad 679 523, Kerala, India. Phone: +91-466 2223038. Email:pa.suresh1 @ gmail.com

Dr.Shyamala K Chengappa (Retired) Professor \& Head of Department Department of Speech Language Pathology All India Institute of Speech \& Hearing Manasagangothri, Mysore 570006 Email: shyamalakc@yahoo.com Phone :9845828913

Dr.Adimoolam Chandrasekhar Research Associate (Physical) Anthropological Survey of India, Southern Regional Centre, ManavBhawan 93/12 \& 94/12, Bogadi,

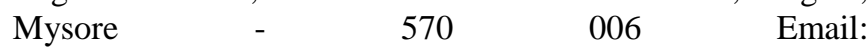
adimoolamchandrasekar@yahoo.com Phone:08277065907

Ms.Ashitha Sidappa Niranjana Murthy Research Scholar Genetics and Genomics Lab, Department of Studies in Genetics and Genomics, University of Mysore, Manasagangotri-06, Karnataka, India Email:ashithasnm09@gmail.com Phone :+91-9739336099

Ms.Dhurbagula Srividhya Senior Research Fellow Department of Speech Language Pathology, All India Institute of Speech \& Hearing Manasagangothri, Mysore 570 006 Email: dsri1987@gmail.com Phone :+91 7022999105 
Sridevi Tirumalaraju Research Scholar -Biotechnology MGNIRSA - (University of Mysore) School of Biotechnology Mahatma Gandhi National Institute of Research and Social Action (MGNIRSA) Kimtee colony, Street No. 1, Rd Number 12, Banjara Hills, Hyderabad, Telangana 500034Email:t_chinni@yahoo.com Phone: 9849964324

Dr.Pardhanandana Reddy Pengaluru Dean, Faculty of Science and Technology, School of Biotechnology, Mahatma Gandhi National Institute of Research and Social Action (MGNIRSA), Kimtee colony, Street No. 1, Rd Number 12, Banjara Hills, Hyderabad, Telangana 500034 Email: reddypp@rediffmail.com Phone: +91-(0)9392434664 